\title{
Evaluación del manejo y disposición final de la gallinaza de reproductora pesada usada como abono orgánico en Costa Rica
}

\author{
Evaluation of the management and final \\ disposal of the poultry litter of heavy breeders \\ used as organic fertilizer in Costa Rica
}

Olger Arce-Solano', Rooel Campos-Rodríguez², Laura Brenes-Peralta ${ }^{3}$

Arce-Solano, O; Campos-Rodríguez, R; Brenes-Peralta, L. Evaluación del manejo y disposición final de la gallinaza de reproductora pesada usada como abono orgánico en Costa

Rica. Tecnología en Marcha. Vol. 33-1. Enero-Marzo 2020.

Pág 165-177.

doi) https://doi.org/10.18845/tm.v33i1.5030

1 Estudiante Maestría en Gestión de Recursos Naturales y Tecnologías de Producción. Instituto Tecnológico de Costa Rica. Costa Rica. Correo electrónico: oarces@dipcmi.co.cr.

2 Doctor en Ciencias Naturales para el Desarrollo Tecnológico de Costa Rica. Correo electrónico: rocampos@tec.ac.cr.

(iD) https://orcid.org/0000-0003-4460-2313

3 Máster en Gerencia y Gestión Ambiental.Tecnológico de Costa Rica. Correo electrónico: labrenes@tec.ac.cr. 


\section{Palabras clave}

Gallinaza; pollinaza; material de cama; abono orgánico.

\section{Resumen}

En Costa Rica hay cinco empresas avícolas que explotan las aves reproductoras pesadas, las cuales son las progenitoras de los pollos de engorde que las mismas empresas producen. Estas aves pasan por dos etapas, la crianza y la etapa de postura y al final de cada etapa generan el residuo conocido como gallinaza. El estudio contempló información de las cinco empresas productoras complementado con datos de importación de las repoductoras obtenidos en el Servicio Nacional de Salud Animal (SENASA), para llegar a determinar que en Costa Rica se produjo en el año 2016 más de 20 mil toneladas métricas de gallinaza.

Después de un proceso de calentamiento en granja, la gallinaza pasa a una bodega hasta su distribución o pasa directamente de la granja a la finca para su uso en cultivos. Durante la investigación del flujo del residuo, se logró la determinación y valoración de Puntos Críticos (PC) los cuales surgieron por desviaciones consideradas en la afectación ambiental, como el uso de gallinaza en suelos agrícolas la cual queda expuesta al ambiente o ligeramente cubierta con tierra que a los días va quedando descubierta.

Al calcular el área agrícola que utiliza gallinaza, se determinó que en el año 2016 fue usada en 2404 hectáreas y de mantener el ritmo de crecimiento de la población de reproductora pesadas, en los próximos 20 años esta área sería duplicada.

El estudio incluye la trazabilidad completa de la gallinaza, desde su origen hasta el uso en fincas agrícolas; por tanto, este trabajo permite la propuesta de mejoras en el manejo y disposición de la gallinaza de reproductoras pesadas usada como abono orgánico en Costa Rica.

\section{Keywords}

Hen manure; chicken manure; bedding material; organic fertilizer.

\section{Abstract}

Five poultry companies raise broiler breeders in Costa Rica. These birds go through two stages, rearing and laying, and at the end of each stage they generate the residue known as manure or litter. This study includes information from the five production companies supplemented with import data obtained from the National Animal Health Service (SENASA), in order to determine that the manure of broiler breeders in Costa Rica amounted more than 20 thousand metric tonnes of litter were produced in 2016.

After a heating process on the chicken house, litter material goes out to a warehouse or passes directly to farms for it to be used in crops as fertilizer. During the research of the residue flow, the determination and evaluation of Critical Points (CP) were obtained, which resulted from deviations considered in the environmental effects, such as the use of poultry in agricultural soils in which the material is exposed to the environment or slightly covered with soil but after some days is uncover.

When calculating the agricultural area that uses poultry manure, it was estimated that 2.404 hectares used this product in 2016, and if the growth rate of the population of broiler breeders is maintained, this area will be doubled within the next 20 years. 
The study includes the complete traceability of this litter material, from its origin to the final application in agricultural farms; therefore, this work allows the proposal of improvements in the management and disposal of the poultry litter of heavy breeders used as organic fertilizer in Costa Rica.

\section{Introducción}

Cada día, el crecimiento de la población demanda más cantidad de alimentos, presentando retos en la producción y productividad agropecuaria, así como en el uso de los recursos naturales [1]. Costa Rica no escapa de dichas proyecciones [2], lo que a su vez ocasiona una mayor generación de residuos [3].

Dadas las condiciones actuales del mercado y la demanda de alimentos, específicamente de los proteicos, la avicultura es la actividad pecuaria que más consumo y crecimiento productivo muestra mundialmente [4]. Particularmente la producción avícola es generadora de residuos orgánicos como la gallinaza, que por muchos años ha sido usada en los campos para el desarrollo y producción de los cultivos. Esta parece ser una buena estrategia que soluciona una necesidad de los agricultores ya que es un material rico en macronutrientes requeridos por las plantas [5], y a su vez permite utilizar un subproducto, contribuyendo mutuamente en un beneficio económico y menor uso de ciertos recursos para obtención de fertilizantes agrícolas.

La producción avícola posee varias modalidades, entre ellas el sistema productivo que comprende a las reproductoras pesadas (gallinas que se cruzan para dar como resultado el pollo de engorde). Una vez terminado su ciclo productivo, se obtiene la gallinaza, la cual es el producto de las deyecciones de las aves junto con el material usado como cama en los galpones avícolas donde estas se mantienen. Normalmente el producto conjunta una serie de materiales usados en el piso del recinto donde están los animales (como granza de arroz, viruta, paja, entre otros), restos de alimento, plumas y las excretas [5]. Por naturaleza, es un producto que contiene material en estado de descomposición, por lo cual debe ser tratado para mitigar los impactos adversos al ambiente de manera que la gallinaza sea convertida en un producto valioso [6].

Ambientalmente es de suma importancia que el uso de la gallinaza como abono orgánico en cultivos agrícolas esté asociado al conocimiento de las necesidades nutricionales del suelo y del cultivo y al valor mineral de la gallinaza [7], de manera que se logre el objetivo de aplicarla una vez procesada, en la cantidad necesaria y que pueda considerarse como un potencial sustituto de fertilizantes químicos, sin que conlleve riesgos a los ecosistemas.

Un inapropiado manejo en cualquier parte del flujo, ya sea en su generación, procesamiento o disposición de la gallinaza, puede llegar a contaminar fuentes de agua, suelos y aire; también es susceptible a generar crecimiento de vectores, malos olores y polvo [5]. Ante una situación de riesgo, los productores avícolas serían sometidos a cuestionamientos por parte de la población y a enfrentar procedimientos legales establecidos por leyes, decretos y reglamentos.

Desde su generación en las granjas avícolas, la gallinaza debe ser manejada adecuadamente cumpliendo con la legislación vigente en el país, la cual responde en términos generales a la Ley para la Gestión Integral de Residuos N8839 [8], que expresamente manifiesta que el generador es responsable del residuo. De manera más específica para estas explotaciones, el Reglamento No 31088-S sobre Granjas Avícolas y el Reglamento sobre el Manejo y control de la Gallinaza y Pollinaza N²9145-MAG-S-MINAE emiten indicaciones para el tratamiento de la gallinaza en sitio o el proceso que finalmente realice el procesador; por su parte el procesador es responsable de la disposición final que tenga el producto [9] [10]. 


\section{Metodología}

\section{Desarrollo del flujo del proceso}

Para llegar a conocer y desarrollar los movimientos de la gallinaza desde la generación en granjas hasta el uso final en fincas agrícolas, fue necesario realizar visitas y consultas durante los meses de agosto, setiembre y octubre del 2017 a granjas avícolas que explotan reproductoras pesadas, así como intermediarios de la gallinaza quienes la sacan de las granjas y fincas que utilizan el residuo avícola. En el caso de las granjas avícolas, fueron consultadas las cinco empresas que trabajan reproductoras pesadas en Costa Rica.

Dentro de los temas de la entrevista se incluyó el volumen que maneja, tratamientos que le dan, embalaje y almacenamiento, tiempo en cada etapa. También fueron consultados asuntos específicos según el lugar, por ejemplo, volumen producido en el caso de las granjas; proceso realizado en el caso de los intermediarios y cantidad aplicada a los cultivos, en las visitas a las fincas.

\section{El flujo y los puntos críticos (PC)}

El análisis del flujo se realizó al consultar y revisar en cada lugar los movimientos que lleva la gallinaza desde que se genera en las granjas de reproductoras, el embalaje y transporte hacia las plantas de proceso y su almacenamiento y uso en las fincas. Para determinar los puntos críticos se contrastó el manejo de la gallinaza en los diversos lugares visitados con información bibliográfica y consultas a especialistas en la materia.

Los PC identificados se analizaron adaptando la herramienta de matrices utilizada para evaluar peligros en la inocuidad de alimentos [11], en donde califica la severidad (S) y la probabilidad de ocurrencia $(\mathrm{O})$, otorgando un valor numérico en la escala del 1 al 5 para cada parámetro, en donde 1 es la calificación más baja sea de severidad o de ocurrencia y 5 la calificación más alta.

Una vez valorado S y O para cada PC, se calculó el Índice de Criticidad (IC), obtenido mediante la fórmula:

Índice de Criticidad (IC) = Severidad (S) x Probabilidad de Ocurrencia (O).

De acuerdo a los datos del IC se valoró la significancia de los PC, para lo cual se usó la escala detallada en el cuadro 1.

Cuadro 1. Parámetros y valores para determinar la significancia de los peligros según el índice de criticidad.

\begin{tabular}{|c|c|}
\hline Significancia & Valor de Significancia \\
\hline Baja & $<10$ \\
\hline Media & $10<X<15$ \\
\hline Alta & $>15$ \\
\hline
\end{tabular}

Fuente: [11].

Producción y área de impacto agrícola de la gallinaza

La producción es estimada con los datos obtenidos en las granjas visitadas, generando un valor promedio por 1.000 aves iniciadas en la etapa de crianza y 1.000 aves iniciadas en la 
etapa de postura. Con el valor anterior y la cantidad de aves que han ingresado al país a través de consultas en el Departamento de Cuarentena Animal del SENASA, se pudo determinar la producción de gallinaza para los años correspondientes a la información obtenida.

\section{Resultados y discusión}

Construcción del flujo de la gallinaza

Una vez que salen las aves de las galeras, se da inicio al tratamiento del material que queda en el piso, al cual se le conoce como la gallinaza. El proceso consiste en provocar un autocalentamiento de la misma conocido como tratamiento de elevación espontánea de la temperatura, uno de los sistemas permitidos según el Reglamento sobre el Manejo y Control de Gallinaza y Pollinaza [10].

Primeramente, se acordona la gallinaza a lo largo de las galeras, como se observa en la figura 1 y luego se procede a taparla con plástico según la figura 2 por un tiempo de tres a cinco días, periodo en donde se mantiene monitoreo de la temperatura. Con este sistema se alcanzan valores entre $55^{\circ} \mathrm{C}$ y $60^{\circ} \mathrm{C}$, los cuales están dentro de lo permitido en el Reglamento indicado para este procedimiento.

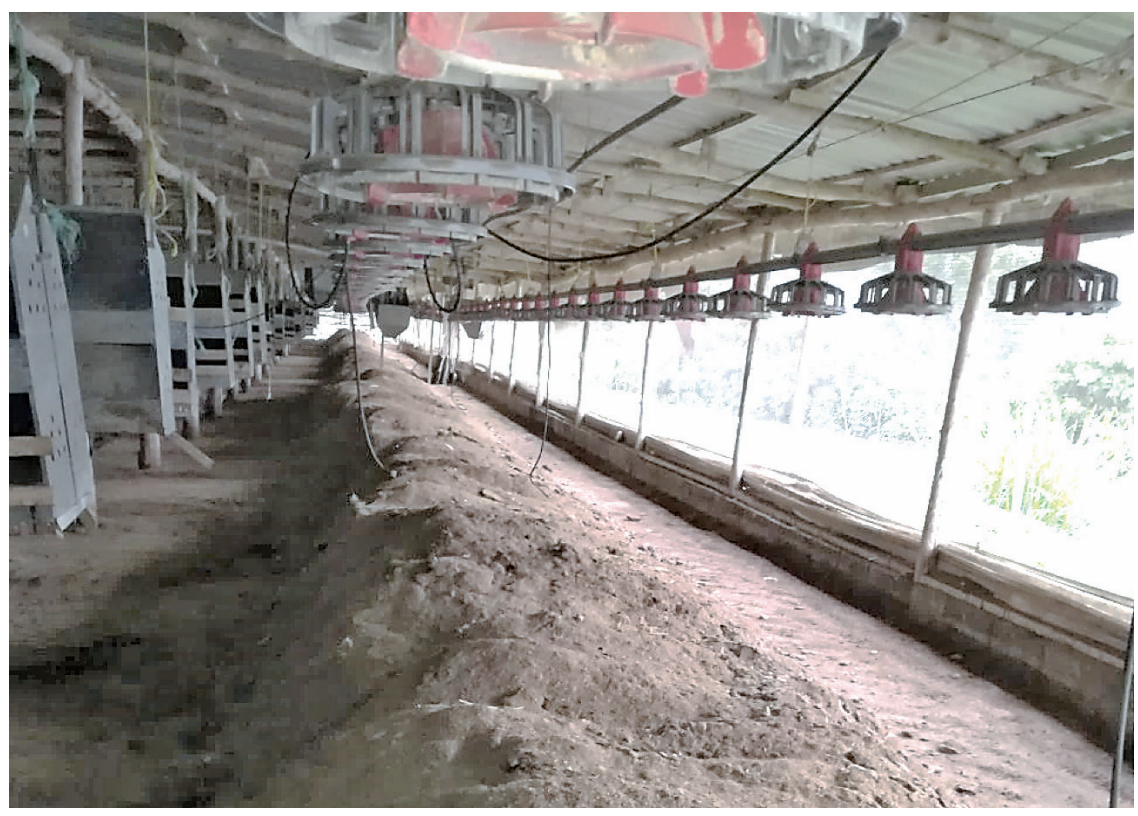

Figura 1. Acordonado de la gallinaza a lo largo de la galera.

El tratamiento de elevación espontánea de la temperatura busca eliminar microorganismos nocivos para las aves de modo que el uso posterior de la gallinaza no se convierta en un riesgo para la salud aviar. En el Reglamento citado se indica que posterior al tratamiento se deberá agregar alguna sustancia o procedimiento contra la proliferación de moscas, mismas que no se observaron en las granjas.

Después del calentamiento se procede a ensacar la gallinaza. En las granjas cuando termina de salir la gallinaza, proceden al lavado y desinfección de las galeras, acarreando los residuos hacia los drenajes existentes. 


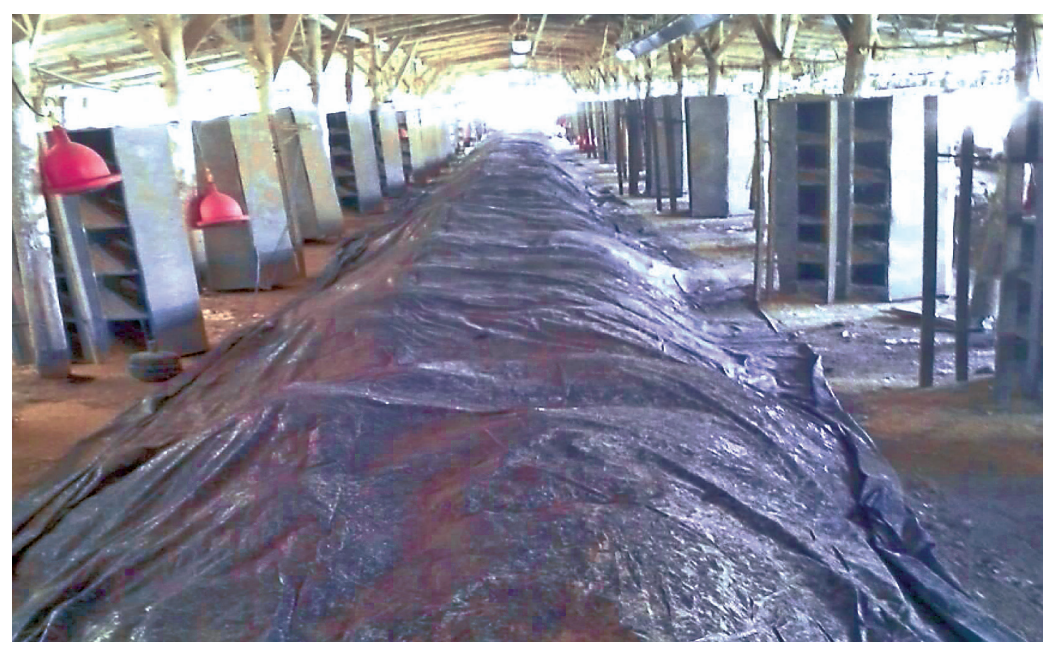

Figura 2. Tapado de la gallinaza acordonada.

La gallinaza puede ser transportada a una bodega para almacenamiento (no es un procesador, sino un intermediario) o directamente a la finca en donde la van a utilizar. En las bodegas del intermediario la gallinaza se almacena con buena ventilación y alta rotación a través de movilización de sacos de un lado a otro para reducir riesgos de incendio, ya que, por la actividad microbiológica, es un producto inflamable.

La figura 3 muestra una bodega donde se almacena gallinaza y en ella se dan los manejos citados.

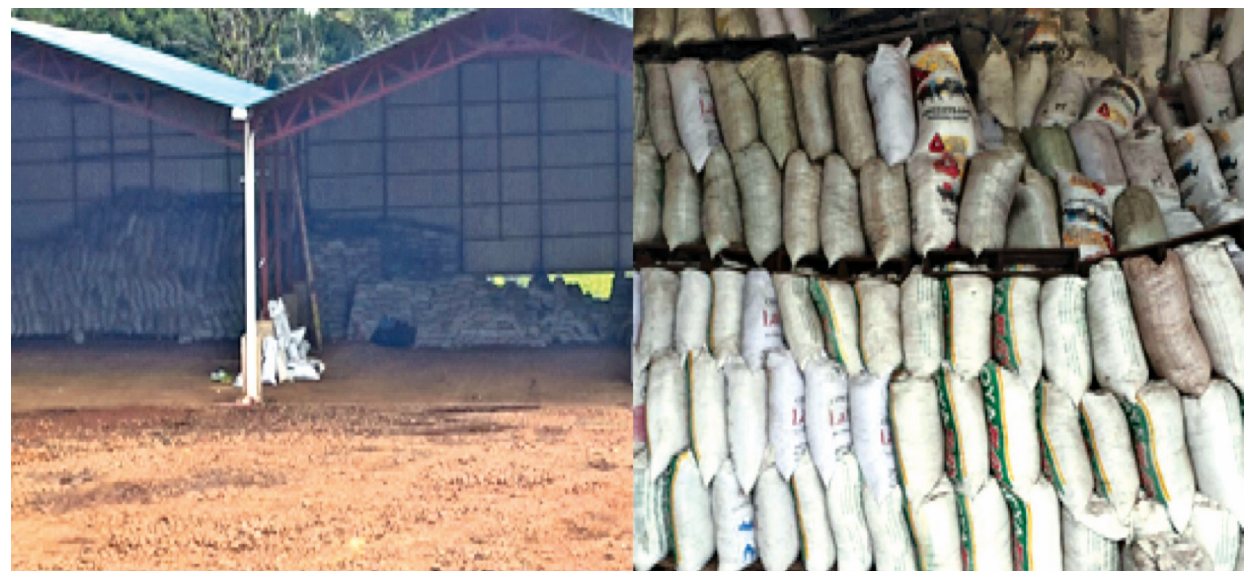

Figura 3. Gallinaza almacenada por un "procesador" en Sabanilla de Alajuela. Izquierda: Bodega, derecha: Forma de estibar los sacos con gallinaza.

La gallinaza que llega a las fincas no ha recibido ningún proceso de fermentación o compostaje, por lo que se considera que es gallinaza cruda. En la finca los agricultores la utilizan en los cultivos tal y como la reciben, sin conocer el aporte nutricional ni las necesidades del suelo o del cultivo. Ocasionalmente cuando la usan la dejan expuesta al suelo y luego proceden a taparla.

La figura 4 presenta el flujo de la gallinaza desde su generación en las granjas de reproductoras pesadas, hasta el uso en las fincas agrícolas, donde un volumen superior al $60 \%$ pasa directo de granja a finca. 


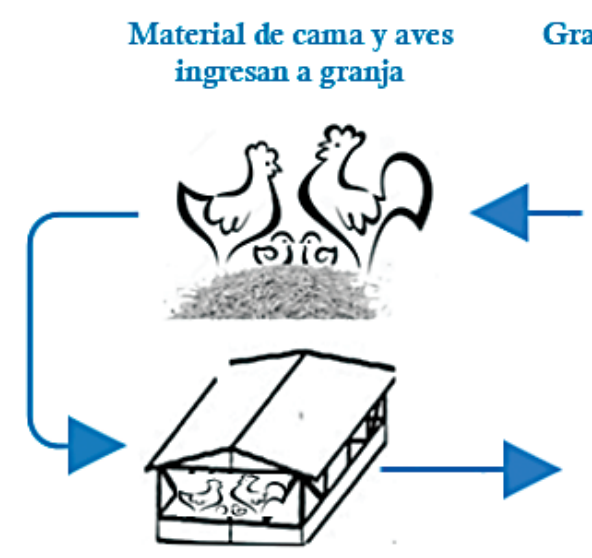

Granja es lavada y residuos en el piso drenados

Granjas prodicen gallinaza cruda

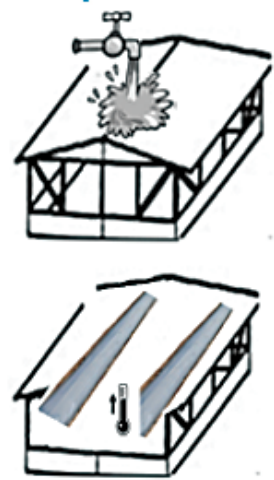

Tratamiento térmico en granja

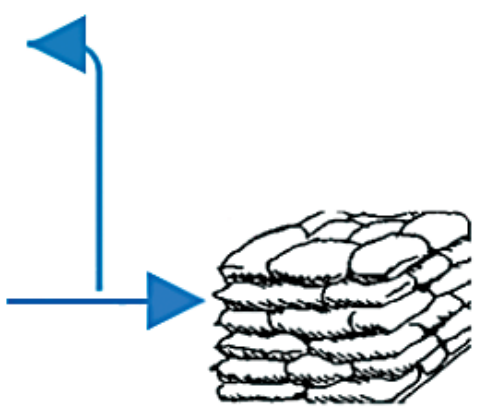

Gallinaza cruda es ensacada

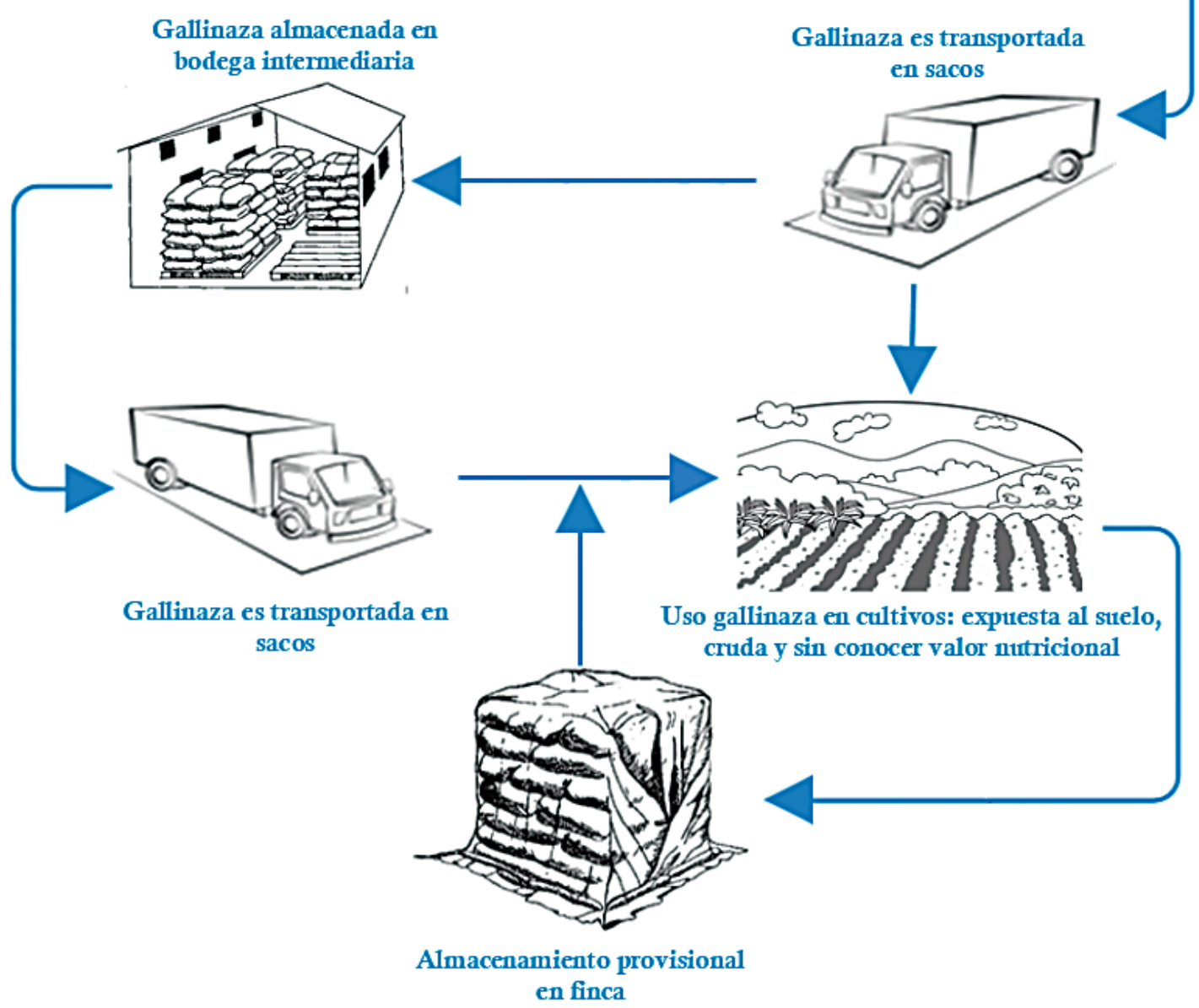

Figura 4. Flujo de la gallinaza con fines agrícolas.

Al consultar los costos generados por la gallinaza desde la granja hasta la finca fue posible generar una estructura de los mismos, presentada en el cuadro 2; el precio de venta de cada saco puesto en la finca del comprador es de $\not 1.200,00$ (un mil doscientos colones) o \$2,09 (dos dólares con nueve centavos). 
Cuadro 2. Estructura de costos de la gallinaza (por saco de 35 kilogramos).

\begin{tabular}{|c|c|c|}
\hline Concepto & Colones $(\phi)$ & Dólares (\$) \\
\hline Gallinaza en granja & 300,00 & 0,52 \\
\hline Saco & 100,00 & 0,17 \\
\hline Ensacado y amarre & 140,00 & 0,24 \\
\hline Transporte granja a bodega & 200,00 & 0,35 \\
\hline Bodegaje & 55,00 & 0,10 \\
\hline Transporte finca (promedio) & 375,00 & 0,65 \\
\hline Total & $1.170,00$ & 2,04 \\
\hline
\end{tabular}

Nota: Tipo de cambio de un dólar ф573,78 del 15 de octubre de 2017 por el Banco Central de Costa Rica.

Ante el bajo margen de utilidad para el intermediario, éste opta por enviar la gallinaza directamente de la granja a la finca, lo que le permite ahorrarse los costos de transporte de granja a bodega y de almacenaje, con lo cual eleva la utilidad a un 23,75\%.

Puntos críticos en la gestión de la gallinaza

El análisis del flujo de la gallinaza puso en evidencia los siguientes puntos críticos respecto a la contaminación ambiental:

Lavado de residuos en granjas. Los residuos que quedan en los pisos de las galeras son lavados y canalizados hacia los drenajes. Estos residuos contienen minerales como Nitrógeno y Fósforo que contaminan aguas superficiales, creando el potencial riesgo de dañar los ecosistemas acuáticos.

Almacenamiento provisional en finca. Cuando la gallinaza es llevada a las fincas, en ocasiones queda producto para uso posterior. El almacenamiento inapropiado de la gallinaza puede causar la generación de moscas y malos olores en el sitio, riesgos de salud a los habitantes de las poblaciones cercanas y contaminar el aire por la producción de gases efecto invernadero.

Gallinaza expuesta usada en cultivos. Partiendo del punto anterior, cuando la gallinaza no queda tapada una vez que se aplica al cultivo, proliferan las moscas y los malos olores por la producción de compuestos volátiles y gases como amoniaco, metano y óxido nitroso, lo que representa un riesgo potencial para las personas que viven en las inmediaciones del lugar y un daño para el medio ambiente.

Uso de gallinaza cruda en cultivos. La legislación nacional no contempla ningún tratamiento adicional a la elevación espontánea de temperatura, solo manifiesta que este puede ser realizado en granja o fuera de ella. Bajo este lineamiento el flujo de la gallinaza es legalmente correcto, pero técnicamente incorrecto, debido a que los nutrientes no se encuentran disponibles de forma inmediata para las plantas y por ello son fácilmente lixiviados al generarse la oxidación del amonio (nitrógeno de la gallinaza) y convertirse en nitrato, contaminando aguas subterráneas [12].

Aparte del uso crudo, los agricultores consultados manifestaron desconocer el aporte nutricional de la gallinaza, así como las condiciones del suelo y las necesidades del cultivo. Ante ello, el uso de gallinaza puede llevar una sobrevaloración nutricional al suelo e impactos ambientales negativos consecuencia de los excedentes empleados. 


\section{Evaluación de los puntos críticos (PC)}

El siguiente cuadro resume la evaluación para cada uno de los PC identificados, en donde se muestra la severidad, la probabilidad de ocurrencia, índice de criticidad y significancia.

Cuadro 3. Matriz de evaluación de los Puntos Críticos.

\begin{tabular}{|c|c|c|c|c|}
\hline Punto Crítico (PC) & $\begin{array}{c}\text { Severidad } \\
\text { (S) }\end{array}$ & $\begin{array}{c}\text { Probabilidad de } \\
\text { Ocurrencia (O) }\end{array}$ & $\begin{array}{c}\text { Índice de } \\
\text { Criticidad (IC) }\end{array}$ & Significancia \\
\hline Lavado de residuos en granjas & 2 & 5 & 10 & Media \\
\hline Almacenamiento provisional en finca & 3 & 3 & 12 & Media \\
\hline Gallinaza expuesta usada en cultivos & 4 & 3 & 25 & Alta \\
\hline Uso de gallinaza cruda en cultivos & 5 & 5 & 9 & Ma \\
\hline
\end{tabular}

Los PC que deben ser atendidos con mayor celeridad por su significancia, son el uso de gallinaza cruda en cultivos y el desconocimiento nutricional de la gallinaza, del suelo y del cultivo por parte de los agricultores. Esta valoración que tiene un factor subjetivo, guarda una estrecha relación con el potencial impacto ambiental negativo que generan ambos riesgos al suelo y a las aguas superficiales y subterráneas.

\section{Cálculo de la producción nacional de gallinaza de reproductoras pesadas}

En el cuadro 4 se presenta el detalle de las cantidades totales de aves reproductoras que fueron importadas al país en el periodo 2012-2016. Estas aves pasan por dos etapas en su ciclo de vida: Desarrollo o crecimiento y reproducción, siendo en la segunda que se producen los huevos para incubar (etapa de postura), de donde nacerán los pollitos de engorde.

Cuadro 4. Importaciones de aves reproductoras pesadas ingresadas a Costa Rica del año 2012 al 2016.

\begin{tabular}{|c|c|}
\hline Año & Aves \\
\hline 2012 & 861.300 \\
\hline 2013 & 833.305 \\
\hline 2014 & 998.547 \\
\hline 2015 & 821.152 \\
\hline 2016 & 1.071 .315 \\
\hline
\end{tabular}

Fuente: [13].

Al consultar en las granjas sobre la mortalidad de las aves, indican que durante la etapa de crianza es de 5\%, por lo que significa una disminución en la etapa de postura, según se muestra en el cuadro 5. 
Cuadro 5. Estimación de aves por ciclo de vida.

\begin{tabular}{|c|c|c|}
\hline Año & Crianza & Postura \\
\hline 2012 & 861.300 & 818.235 \\
\hline 2013 & 833.305 & 791.640 \\
\hline 2014 & 998.547 & 848.620 \\
\hline 2015 & 821.152 & 780.094 \\
\hline 2016 & 1.071 .315 & 1.017 .749 \\
\hline
\end{tabular}

El cuadro 6 detalla la generación de gallinaza de cada empresa que explota aves reproductoras pesadas, expresada en kilogramos por cada un mil aves iniciales en cada etapa de vida.

Cuadro 6. Generación de gallinaza por cada mil aves ingresadas (valores en kilogramos).

\begin{tabular}{|c|c|c|}
\hline Empresa & Crianza & Postura \\
\hline 1 & 8.000 & 13.340 \\
\hline 2 & 6.200 & 11.600 \\
\hline 3 & 7.350 & 12.500 \\
\hline 4 & 5.110 & 15.750 \\
\hline 5 & 7.000 & 13.000 \\
\hline Promedio & 6.732 & 13.238 \\
\hline
\end{tabular}

El promedio de producción de gallinaza multiplicado por la cantidad de aves, permite estimar la producción nacional de gallinaza de reproductoras pesadas durante la crianza y la postura, como se muestra en el cuadro 7 en donde en el año 2016 se generaron más de 20 mil toneladas de gallinaza en Costa Rica.

Cuadro 7. Producción estimada anual de gallinaza de reproductoras pesadas en Costa Rica (en Toneladas Métricas).

\begin{tabular}{|c|c|c|c|}
\hline Año & Crianza & Postura & Total \\
\hline 2012 & 5.798 & 10.832 & 16.630 \\
\hline 2013 & 5.610 & 10.480 & 16.090 \\
\hline 2014 & 6.722 & 11.234 & 17.956 \\
\hline 2015 & 5.528 & 10.327 & 15.855 \\
\hline 2016 & 7.212 & 13.473 & 20.685 \\
\hline
\end{tabular}

\section{Cálculo del área agrícola aplicada con gallinaza}

El uso de la gallinaza en agricultura está muy generalizado en diferentes tipos de cultivos. Una muestra realizada en diversas fincas que usan gallinaza (cuadro 8), permite determinar cantidades entre 4,2 hasta 12,0 toneladas métricas por hectárea (ha). 
Dos agricultores consultados, uno de yuca en Río Cuarto de Alajuela y otro de papa en Capellades de Cartago, justifican el uso de la gallinaza al obtener una mejor productividad, que pasa de un $40 \%$ a 50\%, comparada con fertilizante químico.

No obstante, el uso de la gallinaza en los cultivos tiene un costo económico 3,8 veces mayor que el fertilizante químico, al pagar por esta $\$ 295.000,00$ (doscientos noventa y cinco mil colones) por hectárea mientras que el costo del fertilizante químico es de $\phi 78.000,00$ (setenta y ocho mil colones). Lo anterior sin adicionar el monto de aplicación, el cual es mayor al usar gallinaza que requiere un promedio de 8.600 kilogramos por hectárea contra 300 kilogramos de fertilizante químico.

Cuadro 8. Uso de gallinaza en diversas fincas agrícolas.

\begin{tabular}{|c|c|c|}
\hline Finca & Cultivo & Gallinaza (Tonelada métrica/ha.). \\
\hline 1 & Papa & 8,0 \\
\hline 2 & Café & 12,0 \\
\hline 3 & Pasto & 4,2 \\
\hline 4 & Yuca y piña & 10,0 \\
\hline Promedio & ---- & 8,6 \\
\hline
\end{tabular}

El uso promedio de 8,6 Toneladas métricas/Ha de gallinaza en los cultivos, permite calcular que una producción de gallinaza en el año 2016 de 20.674 Toneladas métricas, se utilizó en más de 2.400 hectáreas. En el cuadro 9 se presenta un estimado de área agrícola en que se usó gallinaza en los últimos cinco años.

Cuadro 9. Estimación de área agrícola que usó gallinaza de reproductoras pesadas del año 2012 al 2016.

\begin{tabular}{|c|c|}
\hline Año & Área (ha.) \\
\hline 2012 & 1.933 \\
\hline 2013 & 1.870 \\
\hline 2014 & 2.086 \\
\hline 2015 & 1.843 \\
\hline 2016 & 2.404 \\
\hline
\end{tabular}

Al tomar como base el año 2012, se tiene un incremento en los últimos cuatro años en el área agrícola que utiliza gallinaza de reproductoras pesadas de 471 hectáreas, con un crecimiento promedio anual de 6,09\%. De mantenerse este ritmo creciente, en el término de 20 años el área agrícola que utiliza este tipo de gallinaza estará duplicada.

Propuestas de mejoras en el manejo y disposición de la gallinaza

Con el fin de reducir los impactos ambientales identificados, se proponen las siguientes mejoras en el manejo y disposición de la gallinaza: 
Gestión en la granja. El tratamiento realizado de elevación espontánea de temperatura a la gallinaza, no es suficiente como proceso de estabilización de un residuo orgánico. Por ello se propone la implementación de un proceso posterior el cual, por tiempo y resguardo de la salud de las aves, debe realizarse fuera de las granjas.

Gestión de los intermediarios. Los intermediarios podrían obtener mayor valor agregado de la gallinaza, ya sea produciendo biogás, compostaje o ambos. El compostaje conlleva una reducción de los microorganismos que pueden competir con los nutrientes de las plantas, minimiza la pérdida de minerales que son causantes de contaminar los ecosistemas, fertiliza y brinda una mejor condición física al suelo y a la vez sustituye o reduce el uso de fertilizantes químicos. Asimismo, ayudará a reducir olores y vectores causantes de molestias en la salud pública.

Gestión en finca. Los agricultores que son los usuarios finales de la gallinaza, requieren que se les brinde mayor información del producto. Se propone para ello la elaboración y colocación en el empaque de instructivos de uso.

Gestión institucional. Los entes rectores designados para el control de la gallinaza, como SENASA, además de los grandes esfuerzos y logros que han alcanzado, deben tener mayor participación en el proceso, especialmente cuando se trata de un residuo producido en grandes cantidades y con impacto ambiental significativo. Su participación debe ser dirigida al control del residuo y a la capacitación a todos los participantes en el flujo. Otros entes como el Ministerio de Agricultura y Ganadería (MAG) pueden participar en la capacitación a procesadores y agricultores en el proceso y uso de la gallinaza como abono orgánico.

\section{Conclusiones}

El análisis del flujo del proceso de la gallinaza permitió confirmar que el manejo de la misma cumple la normativa de aprovechamiento por medio de uno de los tratamientos permitidos que es la elevación espontánea de temperatura, aunque su uso final presenta algunas deficiencias y riesgos al ambiente. La elaboración y estudio del flujo de la gallinaza, puede servir de base para otros procesos productivos que generen residuos de uso agrícola.

Al analizar el flujo completo y determinar los Puntos Críticos, queda en evidencia que la gallinaza constituye un importante problema para la avicultura y otras actividades conexas si no es manejada adecuadamente, ya que es un residuo que se genera en gran volumen y conlleva un potencial impacto negativo, especialmente cuando se usa sin procesar en cultivos en donde está en contacto con el suelo, agua y aire.

Una ventaja que tiene la gallinaza es que se ha convertido en una necesidad para muchos agricultores, lo que facilita la ejecución de mejoras en el procesamiento, ya que los costos adicionados pueden recuperarse en la venta, eso si, tomando en cuenta su costo mayor al fertilizante durante la inversión inicial.

El estudio logró cuantificar que en el año 2016 en Costa Rica la gallinaza producida por la explotación de reproductoras pesadas, fue utilizada en 2.404 hectáreas en cultivos como papa, café, yuca, piña, pastos. Se calcula con el ritmo de producción de esta línea de aves, que el área agrícola que utilizará gallinaza se duplicará en los siguientes 20 años.

La gallinaza de reproductoras pesadas usada como abono orgánico en Costa Rica produce riesgos ambientales y a la salud pública de la forma en que se está usando, por lo que se hace inminente el fortalecimiento de la normativa vigente en procura de la reducción de los impactos ambientales negativos y que los intermediarios se conviertan en procesadores, de manera que al agricultor se le ofrezca un producto mejor procesado. 


\section{Referencias}

[1] Organización para las Naciones Unidas para la Alimentación y la Agricultura, (FAO), "El futuro de la alimentación y la agricultura: Tendencias y desafíos", 2017. [En Línea] Disponible en www.fao.org/3/a-i6583e.pdf [Accedido: 07-ago-2018]

[2] Comisión Económica para América Latina y el Caribe, (CEPAL), "Estimaciones y proyecciones de población a largo plazo 1950-2100", 2017. [En Línea] Disponible en https://www.cepal.org/es/temas/proyeccionesdemograficas/ [Accedido: 09-ago-2018)

[3] M. Ávila, R. Campos, L. Brenes y M. Jiménez. "Generación de biogás a partir del aprovechamiento de residuos sólidos biodegradables en el Tecnológico de Costa Rica, sede Cartago," Tecnología en Marcha, vol. 31, n² pp. 160-170, 2018.

[4] J. Bunge, "La humilde carne de pollo sería la cura para detener el hambre del planeta. Economía y Negocios", 2015. [En Línea] Disponible en http://www.economiaynegocios.cl/ [Accedido: 04-jun-2018]

[5] R.Molaey,A. Bayrakdar, R. Sürmeli y B Çalli. "Anaerobic digestion of chicken manure: Mitigating process inhibition at high ammonia concentrations by selenium supplementation. Biomass and Bioenergy" vol.108, pp. 439-446, 2017.

[6] M. Estrada. "Gestión de la Gallinaza" [En Línea] Disponible en https://albeitar.portalveterinaria.com/noticia/10313/articulos-aves-archivo/gestion-de-la-gallinaza.html [Accedido: 12-jun-2018]

[7] C. Williams. "Gestión de residuos de aves de corral en los países en desarrollo" North Carolina State University, Department of Poultry Science, Raleigh, NC. [En Línea] Disponible en http://www.fao.org/docrep/016/al715s/ al715s00.pdf [Accedido 19-jun-2018]

[8] Asamblea Legislativa Costa Rica, "Ley para la Gestión Integral de Residuos (N 8839)". [En Línea] Disponible en http://www.gaceta.go.cr/pub/2010/07/13/COMP_13_07_2010.html [Accedido: 23-may-2018]

[9] Poder Ejecutivo, "Reglamento sobre granjas avícolas. Decreto Ejecutivo N 31088-S" [En Línea] Disponible en http://www.mag.go.cr/legislacion/2003/de-31088.pdf [Accedido: 09-may-2018]

[10] Poder Ejecutivo, "Reglamento sobre el manejo y control de gallinaza y pollinaza (Decreto N²9145-MAG-SMINAE)" [En Línea] Disponible en http://www.pgrweb.go.cr/scij/Busqueda/Normativa/Normas/nrm_texto_completo.aspx?param1=NRTC\&nValor1=1\&nValor2=45557\&nValor3=47995\&strTipM=TC [Accedido: 8-may-2018]

[11] A. Cartín, A. Villarreal y A. Morera, "Implementación del análisis de riesgo en la industria alimentaria mediante la metodología AMEF: Enfoque práctico y conceptual," Rev. Med. Vet, vol. 27, pp. 133-148, Ene. 2014.

[12] R. Abín, "Impactos ambientales de la producción de huevos: Análisis del ciclo de vida y huella de carbono," Tesis de maestría, Universidad de Oviedo, España, 2016. [13] Dirección de Cuarentena Animal del Servicio Nacional de Salud Animal (Senasa), Importaciones de aves reproductoras pesadas del 2012 al $2016,2017$. 
Tecnología en Marcha,

Vol. 33, N. ${ }^{\circ}$ 1, Enero-Marzo 2020 